\title{
TEAGER ENERGY BASED BLOOD CELL SEGMENTATION
}

\author{
B. Ravi Kumar, Danny K Joseph and T. V. Sreenivas \\ Department of Electrical Communication Engineering, \\ Indian Institute of Science, Bangalore 560 012, India \\ Tel: +91080 3092285; fax: +91 803600563 \\ e-mail: tvsree@ece.iisc.ernet.in
}

\begin{abstract}
A new method for fast and simple segmentation of peripheral blood smears using Teager energy operator (TEO) is presented. The two-pan algorithm efficiently segments leucocytes present in the smear into nucleus and cytoplasm. The local mean weighted high pass filtering property of Teager energy operator is used to identify and distinguish the nucleus of white blood cells. Cytoplasm present in the white blood cells is segmented using selective mathematical morphology. Experimental results are shown for a blood smear containing multiple nnclei. It was observed that modified histogram developed using TEO can efficiently segment even multiple nuclei. Experiments have been conducted to verify the accuracy of the proposed segmentation scheme for all the major types of leucocytes. Simulation results have shown that our algorithm segments nuclei effectively even at low percentage of impulse noise.
\end{abstract}

\section{INTRODUCTION}

The ultimate goal of blood cell segmentation is to extract blood cells from a complicated background and to segment every cell into morphological components such as nucleus, cytoplasm. holes and some others. Cell segmentation is an important and challenging task in medical image processing. The accuracy of segmentation plays a major role in the further steps like leucocyte (white blood cell) identification, classification and automated differential leucocyte count. Numerous segmentation methods for digitized cell image scenes from peripheral blood or bone marrow smears have been proposed. Wermser, et al. [I] introduced a hierarchical scheme using a priori information regarding chromatic propertics of background and cell components. Wu et al. [2] have proposed an Optimal segmentation of cell images, which is robust to noise but can only handle non-overlapping cells, with smooth boundaries or contours. Some limitations of these methods include, use of a priori information and rigid models, requirement of overlapping cells to be dissimilar, [2] etc. We are proposing a two-pan segmentation scheme ${ }^{1}$ that makes use of a discrete time Teager Energy Operator and selective mathematical morphology. Experimental results have shown that our algorithm has the ability to segment majority of the varieties of white blood cells (WBC) correctly. The proposed approach works well in segmenting cytoplasm of WBC by using only luminance component of the image, unlike the conventional approaches that make use of prior knowledge of color information.

\section{SEGMENTATION SCHEME}

In a typical peripheral blood smear color image four regions may be observed which are, namely, the background,

\footnotetext{
${ }^{1}$ This work was partially supported by M/s. Texus Instruments (India) Ltd. as part of their DSP university program.
}

erythrocytes (un-nucleated cells), nucleus and the cytoplasm of the leucocytes. Background is the lighter white pan of the image (highest gray-level), nuclei appear rather dark (lowest gray-level), while erythrocytes display an intermediate level. Other attributes (cytoplasm color, nuclear shape and texture) may differ from one image to the other, depending on the particular type of the cell.

The proposed segmentation scheme shown in Fig. 1 is basically a two-part process of segmenting WBC into nucleus and cytoplasm.

\subsection{Nuclei Segmentation}

Our approach for segmenting nucleus is based on the edges that are effectively detected by Teager energy operator, that has been proposed by Kaiser. One dimensional discrete time TEO of a signal $x(n)$ is defined as

$$
T(n)=x^{2}[n]-x[n+1] . x[n-1]
$$

which has the physical interpretation of total energy for an oscillatory spring mass system. Also, successive samples which are highly correlated are suppressed by this operator. As a result, the edges, which are uncorrelated are expected to stand out separately. TEO can be easily extended to 2 dimensions e.g., Type 1B filter suggested by Mitra [3] which is obtained by applying the filtering operation along the diagonal directions.

$$
\begin{aligned}
T(m, n)= & 2 x^{2}[m, n]-x[m-1, n+1] x[m+1, n-1] \\
& -z[m-1, n-1] x[m+1, n+1]
\end{aligned}
$$

As discussed in [3], the output of Type $1 \mathrm{~B}$ Teager filter is approximately equal to a high-pass filter response weighted by the local-mean $(\boldsymbol{p})$.

$$
\begin{aligned}
& T(m, n) \approx \mu[4 x[m, n]-x[m-1, n+1]- \\
& \quad x[m+1, n-1]-x[m-1, n-1]-x[m+1, n+1]](3)
\end{aligned}
$$




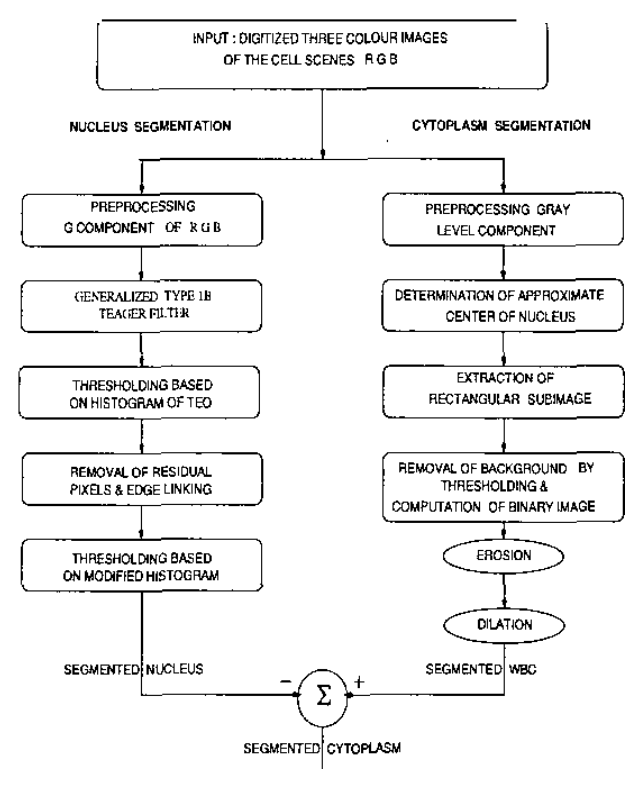

Fig. 1. Block diagram of the proposed two-part segmentation scheme

Type IB filter helps in better identification of edges than other types because most of the peripheral blood smear images are oversampled such that a major portion of the visually sensitive details fall into the midband range. The support of the Type $1 \mathrm{~B}$ filter is larger than that of the Type 1 A filter, whose output is the sum of 2D TEOs evaluated in horizontal and vertical directions. Hence, the passband of the Type $1 \mathrm{~B}$ filter will be close towards the midband region than that of Type $1 \mathrm{~A}$ filter This property has been used for image enhancement \{3\}. It has been shown [4] that the sensitivity of the Type $1 \mathrm{~B}$ Teager filter to the local mean can be adjusted by generalizing TEO by raising each term to a fractional power.

$$
T(n)=x^{2 / m}[n]-[x[n-1] \cdot x[n+1]]^{1 / m}
$$

Like TEO this equation can he approximated as a highpass function weighted by a local mean by applying the binomial theorem

$T(n) \approx \frac{\mu^{(2 / m)-1}}{m}[(x[n]-x[n-1])+(x[n]-x[n+1])]$

The above equation is extended to $2 \mathrm{D}$ thus giving a mean weighted high pass filtering interpretation for generalized Type IB Teager filter .The weighting of the local mean can be adjusted by changing the parameter $\mathrm{m}$. For $\mathbf{m}<\mathbf{2}$, the high-pass output is multiplied by the local mean, raised to some power, and thus is weighted more heavily in high intensity regions. When $m>\mathbf{2}$ the highpass output is divided by the local mean, raised to some power, and thus is weighted more heavily in low intensity regions. For $m=2$, the filter output does not depend on the local mean and approximates the output of the Laplacian filter.

\subsubsection{Algorirhm}

Fig. 1 shows the detailed algorithm for nucleus extraction from the given peripheral blood smear slide. G component of RGB image has been shown to distinguish nuclei from background and combination of cytoplasm and crythrocytes [1] well. Hence, Generalized Type IB Teager filter, which is referred to as Teager filter, with a value of $m>2$ is applied to the $\mathrm{G}$ component of the preprocessed color image so that the edges on the nucleus boundary are highlighted. We have observed that for a particular database the optimum value of $m$ is fairly constant. The threshold for identifying the nucleus boundary is obtained from the histogram plot of Teager filter output. There are isolated pixels, elsewhere in the thresholded Teager filter output mostly due to thresholding and impulse noise present if any. This problem is overcome by using neighborhood rules which remove an isolated pixel if it's number of neighboring pixels in a local window is less than a threshold. Next the nucleus boundary is tracked for discontinuities and an edge linking algorithm is used for filling them. A histogram, corresponding to pixels on the nucleus boundary in the original image identified by the above steps, is plotted. This histogram is clearly bimodal and has a deep valley. But the original histogram lacks this property which makes the threshold selection corresponding to nucleus difficult. The threshold to separate the nucleus is selected from this modified histogram. This threshold selection procedure can he automated.

\subsection{Cytoplasm Segmentation}

Fig. I shows an efficient method of using morphological operations for the segmentation of cytoplasm present in WBC. Morphological operations used here try to differentiate and segment WBC from non interesting objects like RBC based on shape and size. Hence conventional morphological operations fail to segment WBC property when it's size is close to that of cluster of RBCs present in cell e.g., small and medium lymphocytes. Hence, to overcome this problem, location of nucleus identified by above mentioned approach is used, thus segmenting cytoplasm effectively as explained below. Center of each segmented nucleus is determined either by calculating it's center of gravity or by successive morphological erosions by very small structuring element and memorizing the ultimate eroded image. The center of gravity of these ultimate eroded is the center of each nucleus. Background is removed from preprocessed image by simple thresholding $(0.8$ for most of the images) on histogram. All the pixels of objects detected by background removal are assigned gray level 1 while background pixels are assigned 0 level thus obtaining a binary image. A rectangular local window of suitable size centered at calculated center of each nucleus is used to obtain sub-images containing WBC and limited number of RBCs. It has been observed through experiments that problems created by large clusters of RBCs in segmentation of cytoplasm are avoided by this selective local image processing. Mosphological erosion [5], with suitable structuring element, is op. 


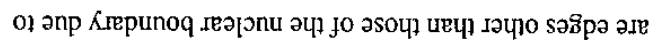

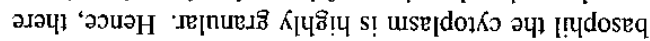

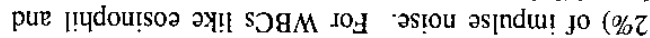

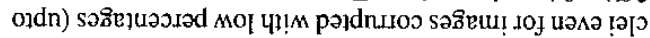

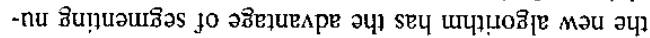

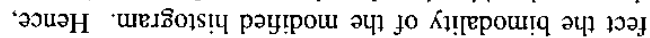

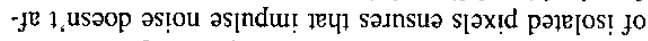

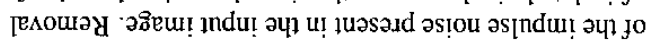

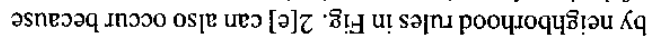

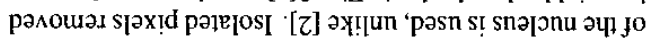

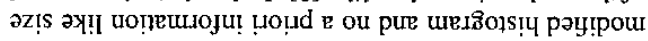

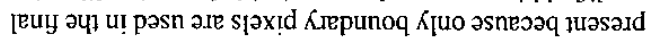

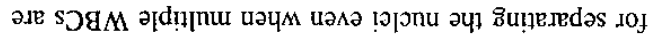

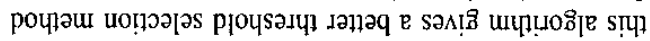
sn५.L [પ]

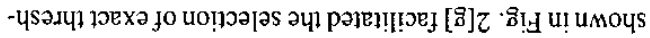

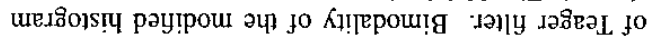

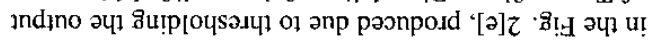

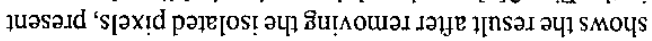
[f]

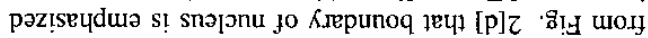

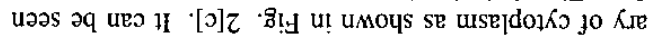

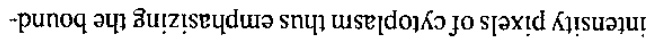

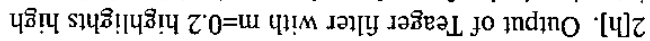

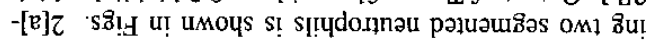

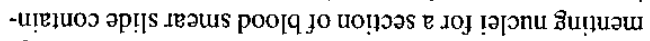

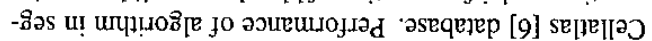

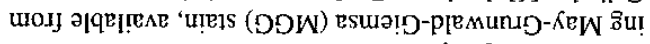

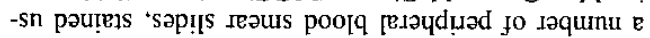

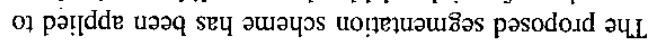

\section{SITISSAY NOILVTANIS $\bullet$}

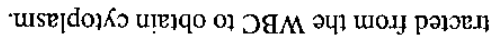

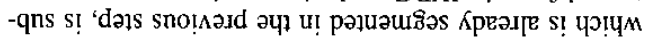

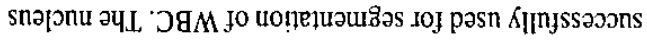

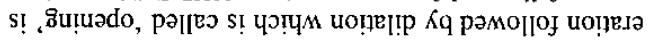

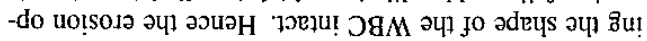

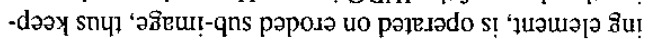

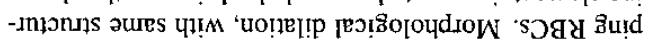

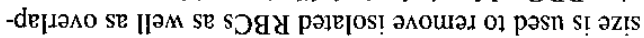

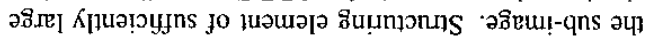

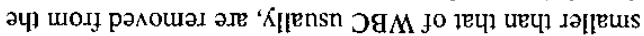

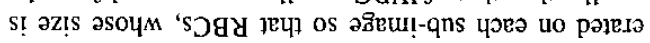

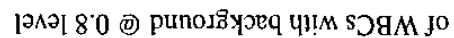

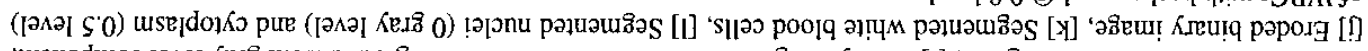

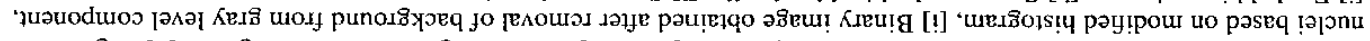

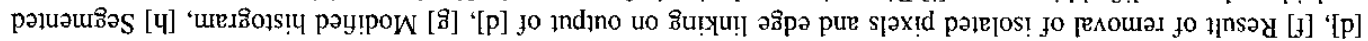
jo ınd’̨no uo su!p

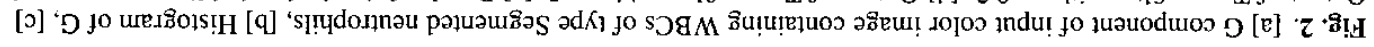

[]]

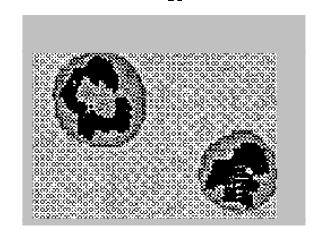

[ᄂ]

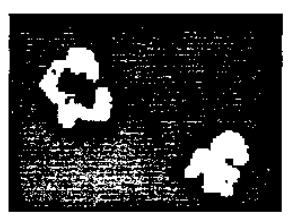

[P]

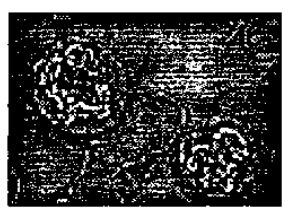

[ㄱ]

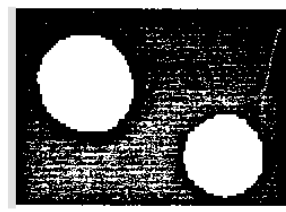

[G]

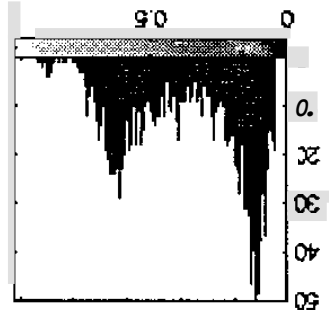

[อ]

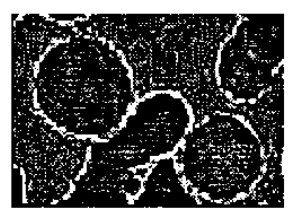

刃]

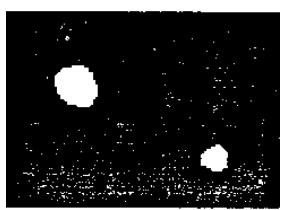

[t]

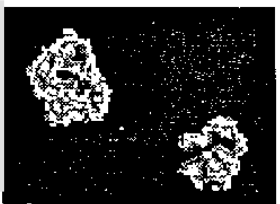

[a]

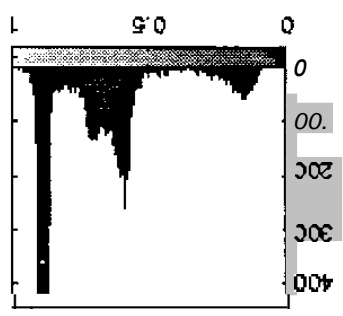

[1]

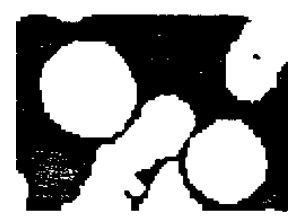

[B]

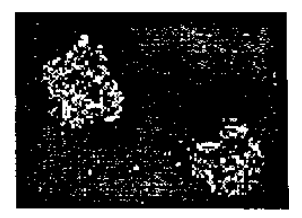

[E]

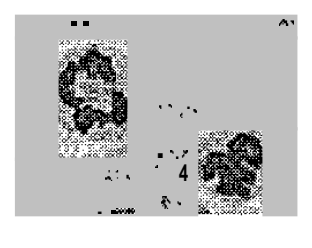



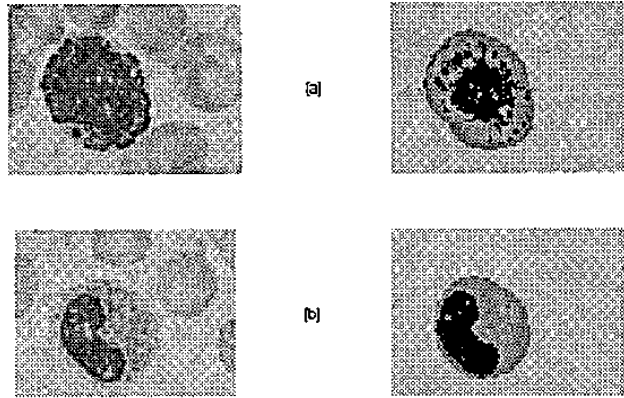

[b]
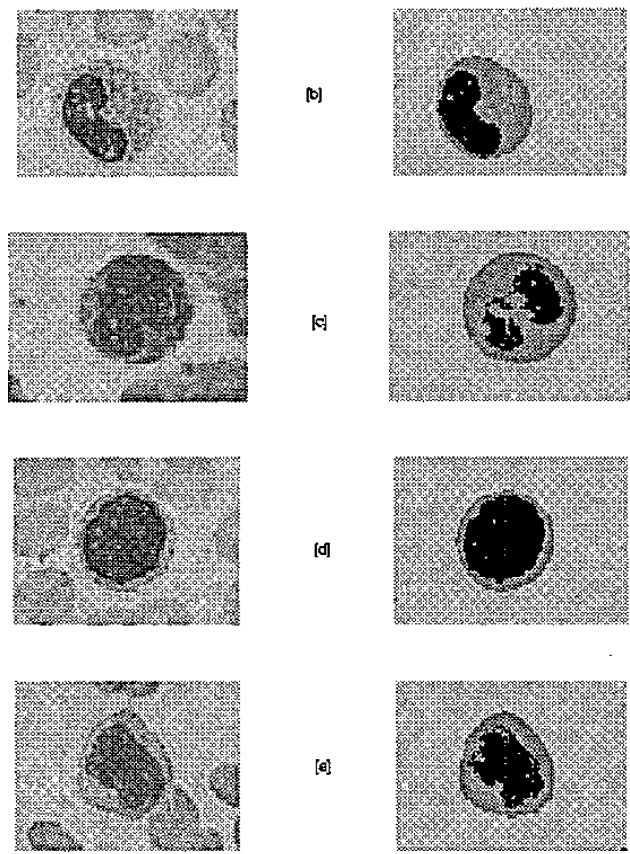

procedure for different types of leucocytes.

Mean weighted high pass filtering analysis of TEO has been made use to select exact threshold for elimination of background also. The samples, of gray image to he segmented, are mapped using a value of $m<2$ $(m=0.2$ here) so that the edges on the cytoplasm border in sub-image are highlighted well as shown in Fig. $2[c]$. It has been observed that similar to nuclei segmentation, here also modified histogram shows two peaks, one for pixels of both $\mathrm{WBC}$ and $\mathrm{RBC}$ and another for background. This bimodal histogram facilitated exact thresholding for eliminating background. An improvement has been done to minimize the distortion caused by morphological operations also. The output of 2 nd part of algorithm is multiplied with TEO of binary image to obtain exact boundary of WBC for non-overlapping cells This boundary is filled to obtain perfectly segmented cytoplasm. This method has been tested with many images but results are not reported here. Even for overlapping cells performance can be improved by applying further morphological operations which is left for future work.

\section{CONCLUSIONS}

We have demonstrated an algorithm for efficient blood cell segmentation using concepts from image processing to isolate individual white blood cells from the given blood smear slide and segment them into nucleus and cytoplasm. Experimental results have shown that our algorithm has the ability to segment the major varieties of WBCs correctly, using only luminance component of the images, unlike the conventional approaches that make use of prior knowledge of color information. The algorithm is found to segment nucleus of WBCs in blood smears correctly even at low levels of impulse noise.

\section{REFERENCES}

[1] D. Wermser, G. Haussman, and C. E. Liedtke, "Segmentation of blood smears by hierarchical thresholding," Computer Vision, Graphics, and Image Processing vol. 25, pp. 151-168, 1984.

[2] H. S. Wu, J, Gil, and J. Barba, "Optimal segmentation of cell images,"Proc. IEE on Vis Image Sig. Processing Vol:145(1), pp. 50-56, 1998

[3] S. K. Mitra, H. Li, I. S. Lin and T. H. Yu, "A new class of nonlinear filters for image enthatice. ment," Proc. IEEE ICASSP, Toronto, Canada, pp $2525-2528,1991$

[4] Michael Moore, Sanjit K. Mitra and Reinhard Bernstein, "A GENERALIZATION OF THE TEAGER ALGORITHM,"Proc. IEEE NSIP 97. Mackinac Island, September 1997.

[5] Anil K. lain, "Fundamentals of Digital Image Processing," Prentice-Hall, New Jersey. 1989.

[6] www.cellatlas.com 